\title{
BANACH SPACE VALUED ORNSTEIN-UHLENBECK PROCESSES \\ INDEXED BY THE CIRCLE
}

\author{
Zdzisław Brzeźniak ${ }^{1}$ \\ Department of Mathematics \\ The University of Hull \\ Hull HU6 7RX, England \\ Z.Brzezniak@maths.hull.ac.uk \\ Jan van Neerven \\ Department of Mathematics \\ Delft University of Technology \\ PO Box 5031, 2600 GA Delft \\ The Netherlands \\ J.vanNeerven@twi.tudelft.nl
}

Abstract - In this paper we study the periodic stochastic abstract Cauchy problem

$$
\begin{aligned}
d X(t) & =A X(t) d t+B d W_{H}(t), \quad t \in[0, T], \\
X(0) & =X(T),
\end{aligned}
$$

where $A$ is the generator of a $C_{0}$-semigroup $\{S(t)\}_{t \geqslant 0}$ on a separable real Banach space, $\left\{W_{H}(t)\right\}_{t \geqslant 0}$ is a suitable cylindrical Wiener process with reproducing kernel Hilbert space $H$, and $B: H \rightarrow E$ is a bounded linear operator. We obtain sufficient conditions for existence of Gaussian mild solutions and show that solutions and compute the covariance of these solutions. We also obtain sufficient conditions which guaratee that the mild solution is law-equivalent with the mild solution at time $T$ of the corresponding stochastic abstract Cauchy problem with zero initial condition.

1991 AMS Subject Classification: 60G15, 60H15, 60B05, 47D03

Running title: Periodic O-U processes

\section{Introduction}

Periodic stochastic processes do appear in many branches of mathematics and mathematical physics. Their importance stems from the fact that traces of certain semigroups are equal to some integrals over periodic maps. For example Bismut [Bi] used such representation to provide a probabilistic prrof of the Atiyah-Singer Theorem. In [ABB] the authors

1 This work was partially supported by EPSRC grant GR/L 60876 . 
have found that a trace of the Schrödinger group can be represented as a Feynman type integral over a certain Hilbert space of periodic maps. Finally, there is much research, see e.g. [JL], towards finding stochastic processes with values in manifold valued periodic maps. This is believed to be an essential step towards proving certain index theorems for Dirac operators, as conjectured by Witten.

In this paper we will be concerned with periodic Ornstein-Uhlenbeck processes (briefly, periodic $\mathrm{O}-\mathrm{U}$ processes). We refer to $[\mathrm{KL}]$ and $[\mathrm{No}]$ for various characterizations of this class of processes. They arise, for instance, in the study of quantum systems in the positive temperature regime are periodic, with their period being inversely proportional to the temperature; see $[\mathrm{KL}],[\mathrm{AH}]$ and references therein.

A standard example of a periodic Ornstein-Uhlenbeck process is the process whose Cameron-Martin space is the Hilbert space $H_{a}^{1,2}\left(S^{1}, \mathbb{R}^{d}\right)$, where $a>0$ is a real number and $S^{1}$ is the unit circle, with norm

$$
\|\gamma\|_{a}^{2}=\int_{0}^{2 \pi}\left(|\dot{\gamma}(s)|^{2}+a^{2}|\gamma(s)|^{2}\right) d s .
$$

It can be shown that this process can be seen, in an appropriate sense, as a solution to the following periodic Itô equation:

$$
\begin{aligned}
d \xi(t) & =-a \xi(t) d t+d w(t), \quad t \in[0,2 \pi] \\
\xi(0) & =\xi(2 \pi)
\end{aligned}
$$

where $w(t)$ is the standard $\mathbb{R}^{d}$-valued Wiener process. Klein and Landau in [KL] have also discussed some infinite-dimensional examples; we also mention the papers [DTb] and [DTd]. It turns out that even such questions as existence and uniqueness are nontrivial and their analysis provides deep insight into other phenomena. In particular, despite its importance, the problem of existence of infinite-dimensional periodic $\mathrm{O}-\mathrm{U}$ processes is not well understood.

In the present paper we study the following periodic Itô equation in infinite dimensions:

$$
\begin{aligned}
d X(t) & =A X(t) d t+B d W_{H}(t), \quad t \in[0, T], \\
X(0) & =X(T) .
\end{aligned}
$$

Here $A$ is the generator of a $C_{0}$-semigroup $\{S(t)\}_{t \geqslant 0}$ on a separable real Banach space $E$, $\left\{W_{H}(t)\right\}_{t \geqslant 0}$ is a suitable cylindrical Wiener process with reproducing kernel Hilbert space $H$, and $B: H \rightarrow E$ is bounded and linear. In fact, the periodic boundary condition is just a special case of more general one of the form $X(0)=\partial X$ for a suitable linear map $\partial: E^{[0, T]} \rightarrow E$, where $E$ is some Banach space, and some of our results can be formulated for this more general setting.

We will describe next our results in the periodic case, i.e. when $\partial X=X(T)$. Under the assumption

$$
I-S(T) \text { is invertible in } E
$$


we prove that (0.1) has a unique solution whenever the corresponding stochastic Cauchy problem

$$
\begin{aligned}
d X(t) & =A X(t) d t+B d W_{H}(t), \quad t \in[0, T], \\
X(0) & =0
\end{aligned}
$$

has a unique solution. In fact, we give an explicit formula that relates the solutions of (0.1) and (0.2). We mention that necessary and sufficient conditions for the unique solvability of (0.2) have been obtained recently in [BN]. We also show that this solution of (0.1) is a centred Gaussian process and we derive an explicit formula for its covariance.

In order to derive the formula relating the solutions of (0.1) and (0.2), in Section 2 we first study the deterministic case. More precisely, we consider the periodic inhomogeneous Cauchy problem

$$
\begin{aligned}
v^{\prime}(t) & =A v(t)+f(t), \quad t \in[0, T], \\
v(0) & =v(T) .
\end{aligned}
$$

As it is well known, under assumption (0.1) this problem has a unique mild solution for every $f \in L^{1}([0, T] ; E)$ which is given explicitly by

$$
u(t)=(I-S(T))^{-1} \int_{0}^{t} S(t-s \bmod T) f(s) d s, \quad t \in[0, T] .
$$

We show that this expression is in fact a special case of a general formula for mild solutions of problems with initial value conditions of the type $v(0)=\partial v$ in terms of the mild solutions of the corresponding Cauchy problem with initial condition $v(0)=0$. As such, this approach is in the spirit of (and to some extent motivated by) the boundary perturbation theory of Greiner [Gr].

\section{A motivating example}

In this section we will perform some computations for the following periodic Itô equation:

$$
\begin{aligned}
d \xi(t) & =-a \xi(t) d t+d w(t), \quad t \in[0,2 \pi], \\
\xi(0) & =\xi(2 \pi),
\end{aligned}
$$

where $a>0$ is a given real number and $\{w(t)\}_{t \in[0,2 \pi]}$ is the standard $\mathbb{R}^{d}$-valued Wiener process. The results of these computations are well-known and will be generalized to infinite dimenstions in Section 3.

Equation (1.1) has a unique weak solution $\left\{\xi_{t}\right\}_{t \in[0,2 \pi]}$; this solution is Gaussian and

its Cameron-Martin space is the Hilbert space $H_{a}^{1,2}\left(S^{1} ; \mathbb{R}^{d}\right)$ of all absolutely continuous functions $\gamma: S^{1} \rightarrow \mathbb{R}^{d}$ such that

$$
\|\gamma\|_{a}^{2}:=\int_{0}^{2 \pi}\left(|\dot{\gamma}(s)|^{2}+a^{2}|\gamma(s)|^{2}\right) d s<\infty .
$$


Starting from the space $H_{a}^{1,2}$, we will give a direct calculation of the covariance of the process $\{\xi(t)\}_{t \in[0,2 \pi]}$. For simplicity of exposition we assume that $d=1$. Given $t \in[0,2 \pi]$ the linear map $H_{a}^{1,2}\left(S^{1} ; \mathbb{R}^{d}\right) \ni \gamma \mapsto \gamma(t) \in \mathbb{R}$ is bounded, hence by the Riesz Representation Theorem there exists a unique $u_{t} \in H_{a}^{1,2}\left(S^{1} ; \mathbb{R}^{d}\right)$ such that

$$
\left[\gamma, u_{t}\right]_{a}=\gamma(t), \quad \gamma \in H_{a}^{1,2}\left(S^{1} ; \mathbb{R}^{d}\right)
$$

Since

$$
\mathbb{E}(\xi(t) \xi(s))=\left[u_{t}, u_{s}\right]_{a}=u_{t}(s)
$$

our task reduces to finding $u_{t}$. Assume now that $t_{0} \in(0,2 \pi)$ is fixed and write $u=u_{t_{0}}$. Then for every $\gamma \in C_{0}^{\infty}\left([0,2 \pi] \backslash\left\{t_{0}\right\}\right)$,

$$
\begin{aligned}
0=\gamma\left(t_{0}\right)=\left[\gamma, u_{t_{0}}\right]_{a} & =\int_{0}^{2 \pi} \dot{u}(s) \dot{\gamma}(s) d s+a^{2} \int_{0}^{2 \pi} u(s) \gamma(s) d s \\
& =\int_{0}^{2 \pi} u(s)\left(-\ddot{\gamma}(s)+a^{2} \gamma(s)\right) d s .
\end{aligned}
$$

Therefore, $u$ is a weak solution to

$$
-\ddot{u}+a^{2} u=0, \text { on }[0,2 \pi] \backslash\left\{t_{0}\right\} .
$$

By the elliptic regularity we have $u \in H^{1,2}\left([0,2 \pi] \backslash\left\{t_{0}\right\}\right)$ and so, in particular, the limits $u\left(t_{0}^{-}\right), u\left(t_{0}^{+}\right), \dot{u}\left(t_{0}^{-}\right), \dot{u}\left(t_{0}^{+}\right)$do all exist. By an integration by parts as above and recalling that $u(0)=u(2 \pi)$ we then infer the following. For any $\gamma \in H_{a}^{2,2}\left(S^{1} ; \mathbb{R}^{d}\right)$,

$$
\int_{0}^{2 \pi} u(s)\left(-\ddot{\gamma}(s)+a^{2} \gamma(s)\right) d s+\left(\dot{u}\left(t_{0}^{-}\right)-\dot{u}\left(t_{0}^{+}\right)\right) \gamma\left(t_{0}\right)=\gamma\left(t_{0}\right) .
$$

Since the map $H_{a}^{1,2}\left(S^{1} ; \mathbb{R}^{d}\right) \ni \gamma \mapsto \gamma\left(t_{0}\right) \in \mathbb{R}$ is onto, we arrive at the following characterization of $u_{t_{0}}$. An element $u \in H^{1,2}\left([0,2 \pi] \backslash\left\{t_{0}\right\}\right)$ equals $u_{t_{0}}$ if and only if it is a weak solution to the boundary value problem

$$
\begin{aligned}
-\ddot{u}+a^{2} u & =0, \text { on }[0,2 \pi] \backslash\left\{t_{0}\right\}, \\
\dot{u}\left(t_{0}^{-}\right)-\dot{u}\left(t_{0}^{+}\right) & =1 \\
u(2 \pi)-u(0) & =0 .
\end{aligned}
$$

For $t_{0}=0$ or $t_{0}=2 \pi$, the problem (1.2) takes the form

$$
\begin{aligned}
-\ddot{u}+a^{2} u & =0, \quad \text { on } \quad(0,2 \pi), \\
\dot{u}(2 \pi)-\dot{u}(0) & =1, \\
u(2 \pi)-u(0) & =0 .
\end{aligned}
$$


Standard but tedious calculations give the following formula for the unique solution $u_{0}$ to (1.3):

$$
\begin{aligned}
u_{0}(s) & =\frac{1}{2 a} \sinh (a s)+\frac{\cosh (\pi a)}{\sinh (\pi a)} \cosh (a s) \\
& =\frac{1}{4 a \sinh ^{2}(\pi a)}[\sinh (a s)+\sinh (a(2 \pi-s))], \quad s \in[0,2 \pi] .
\end{aligned}
$$

The rotation invariance of the scalar product in $H_{a}^{1,2}\left(S^{1} ; \mathbb{R}^{d}\right)$ implies that

$$
u_{t}(s)=u_{0}(s-t \bmod 2 \pi), \quad s \in[0,2 \pi] .
$$

and thus

$$
\mathbb{E}(\xi(t) \xi(s))=\frac{1}{4 a \sinh ^{2}(\pi a)}[\sinh (a(s-t))+\sinh (a(2 \pi-(s-t)))], \quad s, t \in[0,2 \pi] .
$$

In Section 4 we will give a far-reaching generalization of this formula.

\section{The abstract Cauchy problem with an initial value operator}

Throughout this section, $\mathbf{S}=\{S(t)\}_{t \geqslant 0}$ will be a fixed $C_{0}$-semigroup with generator $A$ on a (real or complex) Banach space $E$. We will study the deterministic problem

$$
\begin{aligned}
u^{\prime}(t) & =A u(t)+f(t), \quad t \in[0, T], \\
u(0) & =\partial u,
\end{aligned}
$$

where $f \in L^{1}([0, T] ; E)$, and $\partial: C([0, T] ; E) \rightarrow E$ is a bounded linear operator. Our aim is to give a general formula for the mild solution of this problem in terms of the mild solution of the related inhomogeneous Cauchy problem

$$
\begin{aligned}
u^{\prime}(t) & =A u(t)+f(t), \quad t \in[0, T], \\
u(0) & =0 .
\end{aligned}
$$

We start by defining an operator $L: E \rightarrow C([0, T] ; E)$ by

$$
(L x)(t)=S(t) x, \quad t \in[0, T] .
$$

We let

$$
C_{0}([0, T] ; E):=\{f \in C([0, T] ; E): f(0)=0\}
$$

and for a bounded linear operator $\partial: C([0, T] ; E) \rightarrow E$ we define

$$
C_{\partial}([0, T] ; E)=\{f \in C([0, T] ; E): f(0)=\partial f\} .
$$

Proposition 2.1. For an $f \in C([0, T] ; E)$ the following assertions are equivalent:

(i) $f \in C_{\partial}([0, T] ; E)$;

(ii) $(I-L \partial) f \in C_{0}([0, T] ; E)$.

If $I-L \partial$ is invertible in $C([0, T] ; E)$, then $I-L \partial$ restricts to an isomorphism from $C_{\partial}([0, T] ; E)$ onto $C_{0}([0, T] ; E)$. 
Proof: $(\mathrm{i}) \Rightarrow\left(\right.$ ii): Let $f \in C_{\partial}([0, T] ; E)$, so $f(0)=\partial f$. Then

$$
(I-L \partial) f(0)=f(0)-\operatorname{L\partial f}(0)=f(0)-S(0)(\partial f)=\partial f-\partial f=0
$$

(ii) $\Rightarrow(\mathrm{i})$ : Let $g:=(I-L \partial) f \in C_{0}([0, T] ; E)$, so $g(0)=0$. From

$$
f=(I-L \partial) f+L \partial f=g+L \partial f
$$

we obtain

$$
f(0)=g(0)+\operatorname{L\partial f}(0)=\operatorname{L\partial f}(0)=S(0) \partial f=\partial f
$$

The final assertion is obvious.

A mild solution of $\left(\mathrm{ACP}_{\partial}\right)$ is a function $u \in C_{\partial}([0, T] ; E)$ satisfying

$$
u(t)=S(t) \partial u+\int_{0}^{t} S(t-s) f(s) d s, \quad t \in[0, T]
$$

For $\partial=0$ the problem $(\mathrm{ACP}):=\left(\mathrm{ACP}_{0}\right)$ has a unique mild solution $u$, which is given by

$$
u(t)=\int_{0}^{t} S(t-s) f(s) d s, \quad t \in[0, T]
$$

cf. [Pa, Chapter 4].

Proposition 2.2. Let $f \in L^{1}([0, T] ; E)$ be fixed. Suppose $u$ is a mild solution of $(A C P)$. If $I-L \partial$ is invertible in $C([0, T] ; E)$, then $v=(I-L \partial)^{-1} u$ is a mild solution of $\left(A C P_{\partial}\right)$.

Proof: By assumption we have $u \in C_{0}([0, T] ; E)$ and $u(t)=\int_{0}^{t} S(t-s) f(s) d s$ for all $t \in[0, T]$. Hence $v=(I-L \partial)^{-1} u \in C_{\partial}([0, T] ; E)$ by Proposition 2.1, and for all $t \in[0, T]$ we have

$$
v(t)=L \partial v(t)+(I-L \partial) v(t)=L \partial v(t)+u(t)=S(t) \partial v+\int_{0}^{t} S(t-s) f(s) d s
$$

Let $\Phi: L^{1}([0, T] ; E) \rightarrow C_{0}([0, T] ; E)$ denote the convolution operator defined by

$$
\Phi f(t)=\int_{0}^{t} S(t-s) f(s) d s, \quad t \in[0, T]
$$

If we interpret this operator as the solution operator corresponding to the problem (ACP), then we may regard $(I-L \partial)^{-1} \Phi$ as the solution operator corresponding to $\left(\mathrm{ACP}_{\partial}\right)$. 
Example 2.3. $\quad$ Let $U$ be a bounded linear operator on $E$ commuting with $S(t)$ for all $t \in[0, T]$. In this example we consider the abstract Cauchy problem with holonomy $U$ :

$$
\begin{aligned}
x^{\prime}(t) & =A x(t)+f(t), \quad t \in[0, T], \\
x(0) & =U x(T) .
\end{aligned}
$$

In order to analyze this problem we consider the operator $\partial_{U}: C([0, T] ; E) \rightarrow E$ defined by

$$
\partial_{U} f=U f(T)
$$

We claim: if

$$
I-U S(T) \text { is invertible }
$$

then $I-L \partial_{U}$ is invertible in $C([0, T] ; E)$ and for all $f \in L^{1}([0, T] ; E)$ we have

$$
\left(I-L \partial_{U}\right)^{-1} \Phi f(t)=(I-U S(T))^{-1}\left(\int_{0}^{t} S(t-s) f(s) d s+\int_{t}^{T} U S(T+t-s) f(s) d s\right) .
$$

Once we know this, Proposition 2.2 shows that the right hand side is a mild solution of $\left(\mathrm{ACP}_{U}\right)$.

First we show that $I-L \partial_{U}$ is invertible and that its inverse $\left(I-L \partial_{U}\right)^{-1}$ is given by

$$
\left(I-L \partial_{U}\right)^{-1} g(t)=g(t)+U S(t)(I-U S(T))^{-1} g(T), \quad t \in[0, T], g \in C([0, T] ; E) .
$$

Indeed, the right hand side of this identity defines a bounded operator $J$ on $C([0, T] ; E)$, and for all $g \in C([0, T] ; E)$ we have

$$
\begin{aligned}
& \left(I-L \partial_{U}\right) J g(t) \\
& \quad=\left(I-L \partial_{U}\right)\left(g(\cdot)+U S(\cdot)(I-U S(T))^{-1} g(T)\right)(t) \\
& \quad=\left(g(t)+U S(t)(I-U S(T))^{-1} g(T)\right)-U S(t)\left(g(T)+U S(T)(I-U S(T))^{-1} g(T)\right) \\
& \quad=g(t)
\end{aligned}
$$

and

$$
\begin{aligned}
J\left(\left(I-L \partial_{U}\right) g\right)(t) & =\left(I-L \partial_{U}\right) g(t)+U S(t)(I-U S(T))^{-1}\left(I-L \partial_{U}\right) g(T) \\
& =g(t)-U S(t) g(T)+U S(t)(I-U S(T))^{-1}(g(T)-U S(T) g(T)) \\
& =g(t) .
\end{aligned}
$$

This shows that $J$ is a two-sided inverse of $I-L \partial_{U}$. Next we compute:

$$
\begin{aligned}
\left(I-L \partial_{U}\right)^{-1} \Phi f(t) & =\Phi f(t)+U S(t)(I-U S(T))^{-1} \Phi f(T) \\
& =\int_{0}^{t} S(t-s) f(s) d s+U S(t)(I-U S(T))^{-1} \int_{0}^{T} S(T-s) f(s) d s \\
& =\int_{0}^{t} S(t-s) f(s) d s+U S(T)(I-U S(T))^{-1} \int_{0}^{t} S(t-s) f(s) d s \\
& \quad+(I-U S(T))^{-1} \int_{t}^{T} U S(T+t-s) f(s) d s \\
& =(I-U S(T))^{-1}\left(\int_{0}^{t} S(t-s) f(s) d s+\int_{t}^{T} U S(T+t-s) f(s) d s\right) .
\end{aligned}
$$


The case $U=I$ corresponds to the periodic inhomogeneous Cauchy problem; the above formula then simplifies to

$$
(I-L \partial)^{-1} \Phi f(t)=(I-S(T))^{-1} \int_{0}^{T} S(t-s \bmod T) f(s) d s
$$

here $\partial f=f(T)$ and

$$
t-s \bmod T= \begin{cases}t-s & \text { if } t-s \geqslant 0 \\ T+t-s & \text { if } t-s<0 .\end{cases}
$$

This expression for the mild solution of the periodic Cauchy problem is well known; cf $[\mathrm{Lu}]$.

\section{The periodic stochastic abstract Cauchy problem}

We will use the framework of Section 2 to set up a theory for $E$-valued OrnsteinUhlenbeck processes with holonomy; for this we use the analogy between the linear stochastic Cauchy problem driven by cylindrical noise and the deterministic inhomogenous Cauchy problem. Our terminology is standard; we refer to [DZ], [VTC], [Ne].

It is a well known phenomenon that the paths of an Ornstein-Uhlenbeck process generally fail to be continuous. For this reason the space $C([0, T] ; E)$ may not be appropriate for our discussion, and we have to consider instead the path space $E^{[0, T]}$ consisting of the totality of all $E$-valued functions on $[0, T]$. With respect to the product topology, $E^{[0, T]}$ is a quasi-complete locally convex topological vector space. We let

$$
E_{0}^{[0, T]}:=\left\{f \in E^{[0, T]}: f(0)=0\right\},
$$

and for a continuous linear operator $\partial: E^{[0, T]} \rightarrow E$ we let

$$
E_{\partial}^{[0, T]}:=\left\{f \in E^{[0, T]}: f(0)=\partial f\right\} .
$$

Proposition 3.1. Let $\partial: E^{[0, T]} \rightarrow E$ be a continuous linear operator.

(i) The operator $I-L \partial$ maps $C([0, T]$; E) into itself.

(ii) If $I-L \partial$ is invertible as an operator on $E^{[0, T]}$, then its restriction to $C([0, T] ; E)$ is invertible in $C([0, T] ; E)$.

Proof: (i): For $f \in C([0, T] ; E)$ we have $(I-L \partial) f=f-S(\cdot) \partial f$, which is a continuous function.

(ii): Suppose that $I-L \partial$ is invertible in $E^{[0, T]}$. Fix $f \in C([0, T] ; E)$. Let $g \in E^{[0, T]}$ be such that $f=(I-L \partial) g$. Then

$$
g=(I-L \partial) g+L \partial g=f+S(\cdot) \partial g,
$$

which is a continuous function. This shows that $I-L \partial$ maps $C([0, T] ; E)$ onto itself, and being injective, the restriction of $I-L \partial$ to $C([0, T] ; E)$ is therefore invertible. 
We have the following analogue of Proposition 2.1:

Proposition 3.2. Let $f \in E^{[0, T]}$. The following assertions are equivalent:

(i) $f \in E_{\partial}^{[0, T]}$;

(ii) $(I-L \partial) f \in E_{0}^{[0, T]}$.

If $I-L \partial$ is invertible as an operator on $E^{[0, T]}$, then it restricts to an isomorphism from $E_{\partial}^{[0, T]}$ onto $E_{0}^{[0, T]}$.

Let $H$ be a separable real Hilbert space. A cylindrical Wiener process with reproducing kernel Hilbert space $(R K H S) H$ over a filtered probability space $\left(\Omega, \mathcal{F},\left\{\mathcal{F}_{t}\right\}_{t \in[0, T]}, \mathbb{P}\right)$ is a family of bounded linear operators $\left\{W_{H}(t)\right\}_{t \in[0, T]}$ from $H$ into $L^{2}(\mathbb{P})$ with the following properties:

(i) For all $t \in[0, T]$ and $h \in H, W_{H}(t) h$ is an $\mathcal{F}_{t}$-measurable centred Gaussian random variable;

(ii) For all $h \in H$, the process $\left\{W_{H}(t) h\right\}_{t \in[0, T]}$ is stationary;

(iii) For all $t, s \in[0, T]$ and $h, g \in H$ we have

$$
\mathbb{E}\left(W_{H}(t) h \cdot W_{H}(s) g\right)=(t \wedge s)[h, g]_{H} .
$$

Instead of $W_{H}(t) h$ we will usually write $\left[W_{H}(t), h\right]$.

Note that for all $h \in H$ the real-valued process $\left\{\left[W_{H}(t), h\right]\right\}_{t \in[0, T]}$ is a Brownian motion; in particular it admits a continuous modification.

Throughout the rest of this section $E$ is a separable real Banach space, $H$ is a separable real Hilbert space and $B \in \mathcal{L}(H, E)$ is a bounded linear operator. Let $\partial: E^{[0, T]} \rightarrow E$ be a continuous linear operator. We consider the stochastic abstract Cauchy problem

$$
\begin{aligned}
d X_{t} & =A X_{t} d t+B d W_{H}(t), \quad t \in[0, T] \\
X_{0} & =\partial X
\end{aligned}
$$

where $\left\{W_{H}(t)\right\}_{t \in[0, T]}$ is a cylindical Wiener process with RKHS H. A weak solution is an $E$-valued stochastic process $\left\{X_{t}\right\}_{t \in[0, T]}$ such that for all $x^{*} \in D\left(A^{*}\right), t \mapsto\left\langle A^{*} x^{*}, X_{t}\right\rangle$ is integrable almost surely and

$$
\left\langle X_{t}, x^{*}\right\rangle=\left\langle\partial X, x^{*}\right\rangle+\int_{0}^{t}\left\langle X_{s}, A^{*} x^{*}\right\rangle d s+\left[W_{H}(t), B^{*} x^{*}\right]
$$

almost surely. Here the random variable $\partial X$ is defined by

$$
(\partial X)(\omega)=\partial(X(\omega))
$$

where $X(\omega) \in E^{[0, T]}$ is given by $(X(\omega))(t)=X_{t}(\omega)$.

Note that if $\left\{X_{t}\right\}_{t \in[0, T]}$ is a weak solution, then by (3.1), $\left\langle\partial X, x^{*}\right\rangle$ is a measurable function for each $x^{*} \in D\left(A^{*}\right)$, and hence, by an approximation argument, for each $x^{*} \in E^{*}$. Since $\partial X$ is also separably valued, it follows that $\partial X$ is strongly measurable. Unless $\partial=0$, because of the initial condition it makes no sense to impose predictability of $\left\{X_{t}\right\}_{t \in[0, T]}$.

If $\partial=0$ we refer to the avove problem as (sACP). Necesary and sufficent conditions for the existence of a predictable weak solution of ( $\mathrm{sACP}$ ) are given in $[\mathrm{BN}]$; for the convenience of the reader we state the result. 
Theorem 3.3. The following assertions are equivalent:

(i) The problem $(A C P)$ has a predictable $E$-valued weak solution $\left\{X_{t}\right\}_{t \in[0, T]}$ on $[0, T]$;

(ii) The operator $Q_{T} \in \mathcal{L}\left(E^{*}, E\right)$ defined by

$$
Q_{T} x^{*}:=\int_{0}^{T} S(t) B B^{*} S^{*}(t) x^{*} d t, \quad x^{*} \in E^{*},
$$

is the covariance operator of a centred Gaussian Borel measure on E.

In this situation, the solution $\left\{X_{t}\right\}_{t \in[0, T]}$ is unique, and satisfies

$$
\left\langle X_{t}, x^{*}\right\rangle=\int_{0}^{t}\left\langle S(t-s) \circ B d W_{H}(s), x^{*}\right\rangle, \quad t \in[0, T], x^{*} \in E^{*} .
$$

The process $\left\{X_{t}\right\}_{t \in[0, T]}$ is an Ornstein-Uhlenbeck process with drift $A$ and forcing operator $B$, i.e. a centred Gaussian process with covariance

$$
\mathbb{E}\left(\left\langle X_{t}, x^{*}\right\rangle\left\langle X_{s}, y^{*}\right\rangle\right)=\int_{0}^{t \wedge s}\left[B^{*} S^{*}(t-u) x^{*}, B^{*} S^{*}(s-u) y^{*}\right]_{H} d u
$$

for all $x^{*}, y^{*} \in E^{*}$ and $0 \leqslant t, s \leqslant T$. It has a version with almost surely square integrable trajectories. If the semigroup generated by $A$ is analytic, then $\left\{X_{t}\right\}_{t \in[0, T]}$ has a version with continuous trajectories.

Parallel to Proposition 2.2 we have the following result:

Theorem 3.4. Let $\left\{X_{t}\right\}_{t \in[0, T]}$ be a weak solution of the problem

$$
\begin{aligned}
d X_{t} & =A X_{t} d t+B d W_{H}(t), \quad t \in[0, T], \\
X_{0} & =0
\end{aligned}
$$

Let $\partial: E^{[0, T]} \rightarrow E$ be a continuous linear operator such that $I-L \partial$ is invertible in $E^{[0, T]}$. Then the Gaussian process $\left\{Y_{t}\right\}_{t \in[0, T]}$, where $Y_{t}=\left((I-L \partial)^{-1} X\right)_{t}$, is a weak solution of the problem

$$
\begin{aligned}
d Y_{t} & =A Y_{t} d t+B d W_{H}(t), \quad t \in[0, T], \\
Y_{0} & =\partial Y .
\end{aligned}
$$

Proof: As was stated in Theorem 3.3, the weak solution $\left\{X_{t}\right\}_{t \in[0, T]}$ of (sACP) are almost surely square integrable:

$$
\int_{0}^{t}\left\|X_{t}(\omega)\right\|^{2} d t<\infty \quad \text { for almost all } \omega \in \Omega
$$

It follows that also the process $\left\{Y_{t}\right\}_{t \in[0, T]}$ is almost surely square integrable. Hence if $x^{*} \in D\left(A^{*}\right)$ is given, the map $t \mapsto\left\langle Y_{t}, A^{*} x^{*}\right\rangle$ is integrable almost surely. For almost all $\omega \in \Omega$ we have

$$
\left((I-L \partial)(Y(\omega))(t)=X_{t}(\omega)=(X(\omega))(t)=(I-L \partial)(X(\omega))(t)+L \partial(X(\omega))(t) .\right.
$$


Multiplying both sides with $(I-L \partial)^{-1}$, we obtain

$$
Y_{t}=X_{t}+L \partial(Y(\omega))=X_{t}+S(t) \partial Y
$$

Using this, we compute

$$
\begin{aligned}
\int_{0}^{t}\left\langle Y_{s}, A^{*} x^{*}\right\rangle d s & =\int_{0}^{t}\left\langle X_{s}, A^{*} x^{*}\right\rangle d s+\int_{0}^{t}\left\langle S(s) \partial Y, A^{*} x^{*}\right\rangle d s \\
& =\int_{0}^{t}\left\langle X_{s}, A^{*} x^{*}\right\rangle d s+\left\langle S(t) \partial Y-\partial Y, x^{*}\right\rangle .
\end{aligned}
$$

Hence,

$$
\begin{aligned}
\left\langle Y_{t}, x^{*}\right\rangle & -\left\langle\partial Y, x^{*}\right\rangle-\int_{0}^{t}\left\langle Y_{s}, A^{*} x^{*}\right\rangle d s \\
& =\left(\left\langle X_{t}, x^{*}\right\rangle+\left\langle S(t) \partial Y, x^{*}\right\rangle\right)-\left\langle\partial Y, x^{*}\right\rangle-\int_{0}^{t}\left\langle X_{s}, A^{*} x^{*}\right\rangle d s-\left\langle S(t) \partial Y-\partial Y, x^{*}\right\rangle \\
& =\left\langle X_{t}, x^{*}\right\rangle-\int_{0}^{t}\left\langle X_{s}, A^{*} x^{*}\right\rangle d s \\
& =\left[W_{H}(t), B^{*} x^{*}\right] .
\end{aligned}
$$

Define the continuous linear map $\Phi_{H}: L^{2}([0, T] ; H) \rightarrow E^{[0, T]}$ by

$$
\Phi_{H} f(t):=\int_{s}^{t} S(t-s) B f(s) d s .
$$

We will also interpret $L$ as an operator from $E$ into $E^{[0, T]}$. In order to stress that the range space is the locally convex space $E^{[0, T]}$, the continuous adjoints of $\Phi_{H}$ and $L$ will be denoted by $\Phi_{H}^{\prime}$ and $L^{\prime}$, respectively.

Lemma 3.5. We have

$$
\Phi_{H}^{\prime}\left(\delta_{t} \otimes x^{*}\right)=\chi_{[0, t]}(\cdot) B^{*} S^{*}(t-\cdot) x^{*} .
$$

Proof: For all $f \in L^{2}([0, T] ; H)$ we have

$$
\begin{aligned}
\left\langle\Phi_{H} f, \delta_{t} \otimes x^{*}\right\rangle & =\int_{0}^{t}\left\langle S(t-s) B f(s), x^{*}\right\rangle d s \\
& =\int_{0}^{t}\left[f(s), B^{*} S^{*}(t-s)\right]_{H} d s \\
& =\left[f, \chi_{[0, t]}(\cdot) B^{*} S^{*}(t-\cdot) x^{*}\right]_{L^{2}([0, T] ; H)} .
\end{aligned}
$$


Thanks to this lemma, the covariance of an Ornstein-Uhlenbeck process can be rewritten in terms of $\Phi_{H}$ as follows:

$$
\mathbb{E}\left(\left\langle X_{t}, x^{*}\right\rangle\left\langle X_{s}, y^{*}\right\rangle\right)=\left[\Phi_{H}^{\prime}\left(\delta_{t} \otimes x^{*}\right), \Phi_{H}^{\prime}\left(\delta_{s} \otimes y^{*}\right)\right]_{L^{2}([0, T] ; H)}
$$

Theorem 3.6. Let $X=\left\{X_{t}\right\}_{t \in[0, T]}$ be a weak solution of (sACP) and let

$$
Y=(I-L \partial)^{-1} X
$$

be the corresponding weak solution of $\left(\mathrm{sACP}_{\partial}\right)$. Then for all $x^{*}, y^{*} \in E^{*}$ and all $0 \leqslant t, s \leqslant$ $T$ we have

$$
\mathbb{E}\left(\left\langle Y_{t}, x^{*}\right\rangle\left\langle Y_{s}, y^{*}\right\rangle\right)=\left[\Phi_{H}^{\prime}\left(I-\partial^{\prime} L^{\prime}\right)^{-1}\left(\delta_{t} \otimes x^{*}\right), \Phi_{H}^{\prime}\left(I-\partial^{\prime} L^{\prime}\right)^{-1}\left(\delta_{s} \otimes y^{*}\right)\right]_{L^{2}([0, T] ; H)}
$$

Proof: We can reformulate (3.3) by saying that

$$
\left[\left\langle X(\cdot), \delta_{t} \otimes x^{*}\right\rangle,\left\langle X(\cdot), \delta_{s} \otimes y^{*}\right\rangle\right]_{L^{2}(\mathbb{P})}=\left[\Phi_{H}^{\prime}\left(\delta_{t} \otimes x^{*}\right), \Phi_{H}^{\prime}\left(\delta_{s} \otimes y^{*}\right)\right]_{L^{2}([0, T] ; H)} .
$$

It is a routine exercise to check that every element in the topological dual $\left(E^{[0, T]}\right)^{\prime}$ of $E^{[0, T]}$ can be represented as a finite linear combination of functionals of the form $\delta_{t} \otimes x^{*}$ with $t \in[0, T]$ and $x^{*} \in E^{*}$. By bilinearity, it follows from (3.4) that

$$
[\langle X(\cdot), \phi\rangle,\langle X(\cdot), \psi\rangle]_{L^{2}(\mathbb{P})}=\left[\Phi_{H}^{\prime}(\phi), \Phi_{H}^{\prime}(\psi)\right]_{L^{2}([0, T] ; H)}, \quad \forall \phi, \psi \in\left(E^{[0, T]}\right)^{\prime} .
$$

Using this, we compute

$$
\begin{aligned}
\mathbb{E}\left(\left\langle Y_{t}, x^{*}\right\rangle\left\langle Y_{s}, y^{*}\right\rangle\right) & =\left[\left\langle Y(\cdot), \delta_{t} \otimes x^{*}\right\rangle,\left\langle Y(\cdot), \delta_{s} \otimes y^{*}\right\rangle\right]_{L^{2}(\mathbb{P})} \\
& =\left[\left\langle(I-L \partial)^{-1} X(\cdot), \delta_{t} \otimes x^{*}\right\rangle,\left\langle(I-L \partial)^{-1} X(\cdot), \delta_{s} \otimes y^{*}\right\rangle\right]_{L^{2}(\mathbb{P})} \\
& =\left[\left\langle X(\cdot),\left(I-\partial^{\prime} L^{\prime}\right)^{-1}\left(\delta_{t} \otimes x^{*}\right)\right\rangle,\left\langle X(\cdot),\left(I-\partial^{\prime} L^{\prime}\right)^{-1}\left(\delta_{s} \otimes y^{*}\right)\right\rangle\right]_{L^{2}(\mathbb{P})} \\
& =\left[\Phi_{H}^{\prime}\left(\left(I-\partial^{\prime} L^{\prime}\right)^{-1}\left(\delta_{t} \otimes x^{*}\right)\right), \Phi_{H}^{\prime}\left(\left(I-\partial^{\prime} L^{\prime}\right)^{-1}\left(\delta_{s} \otimes y^{*}\right)\right)\right]_{L^{2}([0, T] ; H)} .
\end{aligned}
$$

An $E$-valued centred Gaussian process whose covariance is given by (3.3) will be called a $\partial$-Ornstein-Uhlenbeck process with drift $A$ and forcing operator $B \in \mathcal{L}(H, E)$.

Example 3.7. We will work out the formula for an Ornstein-Uhlenbeck process with holonomy $U$, i.e. an $\partial$-Ornstein-Uhlenbeck process with $\partial=\partial_{U}$ given by $\partial_{U} f=U f(T)$ as in Example 2.3. As before, we assume that $U$ is a bounded operator on $E$ commuting with each $S(t)$.

Arguing as in Example 2.3, we see that if $I-U S(T)$ is invertible, then the operator $I-L \partial_{U}$ is invertible in $E^{[0, T]}$ and its inverse is given by

$$
\left(I-L \partial_{U}\right)^{-1} g(t)=g(t)+U S(t)(I-U S(T))^{-1} g(T), \quad g \in E^{[0, T]} .
$$


For $g \in E^{[0, T]}$ we compute:

$$
\begin{aligned}
\left\langle\left(I-L \partial_{U}\right)^{-1} g, \delta_{t} \otimes x^{*}\right\rangle & =\left\langle g(\cdot)+U S(\cdot)(I-U S(T))^{-1} g(T), \delta_{t} \otimes x^{*}\right\rangle \\
& =\left\langle g(t)+U S(t)(I-U S(T))^{-1} g(T), x^{*}\right\rangle \\
& =\left\langle g,\left(\delta_{t} \otimes x^{*}+\delta_{T} \otimes U^{*} S^{*}(t)\left(I-U^{*} S^{*}(T)\right)^{-1} x^{*}\right)\right\rangle .
\end{aligned}
$$

From this we see that

$$
\left(I-\partial_{U}^{\prime} L^{\prime}\right)^{-1}\left(\delta_{t} \otimes x^{*}\right)=\delta_{t} \otimes x^{*}+\delta_{T} \otimes\left(U^{*} S^{*}(t)\left(I-U^{*} S^{*}(T)\right)^{-1} x^{*}\right.
$$

and hence,

$$
\begin{aligned}
\Phi_{H}^{\prime}(I- & \left.\partial_{U}^{\prime} L^{\prime}\right)^{-1}\left(\delta_{t} \otimes x^{*}\right) \\
& =\Phi_{H}^{\prime}\left(\delta_{t} \otimes x^{*}+\delta_{T} \otimes\left(U^{*} S^{*}(t)\left(I-U^{*} S^{*}(T)\right)^{-1} x^{*}\right)\right. \\
& =\chi_{[0, t]}(\cdot) B^{*} S^{*}(t-\cdot) x^{*}+B^{*} U^{*} S^{*}(T-\cdot) S^{*}(t)\left(I-U^{*} S^{*}(T)\right)^{-1} x^{*} \\
& =B^{*}\left(I-U^{*} S^{*}(T)\right)^{-1}\left(\chi_{[0, t]}(\cdot) S^{*}(t-\cdot) x^{*}+\chi_{[t, T]}(\cdot) U^{*} S^{*}(T+t-\cdot) x^{*}\right) .
\end{aligned}
$$

Note the similarity with the formula obtained in Example 2.3. In combination with (3.3), this gives the covariance of an Ornstein-Uhlenbeck process with holonomy $U$.

Let us now take a look at the periodic case $U=I$. For the semigroup $S(t)=e^{-a t} I$, with $a>0$ a given real number, the problem $\left(\mathrm{sACP}_{\partial}\right)$ reduces to $(1.1)$. We can compute the covariance of its weak solution $\left\{Y_{t}\right\}_{t \in[0, T]}$ explicity: from (3.3) and (3.5) we obtain after an elementary computation that

$$
\mathbb{E}\left(\left\langle Y_{t}, x^{*}\right\rangle\left\langle Y_{s}, y^{*}\right\rangle\right)=\frac{1}{4 a \sinh ^{2}\left(\frac{a T}{2}\right)}(\sinh (a(t-s))+\sinh (a(T-(t-s))))\left[B^{*} x^{*}, B^{*} y^{*}\right]_{H} .
$$

This is exactly the formula (1.4) derived in Section 1, where we took $T=2 \pi$.

The case $U=-I$ in this example corresponds to the 'twisted' case, which we discuss briefly next.

Example 3.8. For $U=-I$ and $S(t)=e^{-a t} I$ with $a>0$, a comutation similar to the one above gives the following formula for the covariance:

$$
\begin{aligned}
\mathbb{E}\left(\left\langle Y_{t}, x^{*}\right\rangle\left\langle Y_{s}, y^{*}\right\rangle\right) & \\
& =\frac{1}{4 a \cosh ^{2}\left(\frac{a T}{2}\right)}(-\sinh (a(t-s))+\sinh (a(T-(t-s))))\left[B^{*} x^{*}, B^{*} y^{*}\right]_{H} .
\end{aligned}
$$

In the present case, we may take any $a \in \mathbb{R}$; with $a=0$ the resulting covariance for the resulting 'twisted Brownian bridge' becomes

$$
\mathbb{E}\left(\left\langle Y_{t}, x^{*}\right\rangle\left\langle Y_{s}, y^{*}\right\rangle\right)=\frac{T-2(t-s)}{4}\left[B^{*} x^{*}, B^{*} y^{*}\right]_{H}
$$




\section{The periodic problem versus the Cauchy problem: law equiv- alence}

In this final section we suppose again that $E$ is a separable real Banach space and that $A$ is a generator of a $C_{0}$-semigroup $\{S(t)\}_{t \geqslant 0}$ on $E$ with generator $A$. Our aim in this section is to compare the laws of the periodic O-U process $\left\{Y_{t}\right\}_{t \in[0, T]}$ with those of the O-U process $\left\{X_{t}\right\}_{t \in[0, T]}$ corresponding to the Cauchy problem with initial value operator $\partial=0$ (cf. the notation used in Theorem 3.3). Throughout this section we assume that $I-S(T)$ is invertible.

The laws of the random variables $Y_{t}$ en $X_{t}$ will be denoted by $\nu_{t}$ and $\mu_{t}$, respectively; these are centred Gaussian Borel measures on $E$. By $H_{t}$ and $G_{t}$ we denote the reproducing kernel Hilbert spaces of $\nu_{t}$ and $\mu_{t}$, respectively, and by $R_{t}$ and $Q_{t}$ their covariance operators.

Lemma 4.1. For all $t \in[0, T]$ we have $R_{t}=R_{T}=(I-S(T))^{-1} Q_{T}\left(I-S^{*}(T)\right)^{-1}$.

Proof: For all $t \in[0, T]$ and $x^{*} \in E^{*}$ we have

$$
\begin{aligned}
\left\langle R_{t} x^{*}, x^{*}\right\rangle=\left\|R_{t} x^{*}\right\|_{H_{t}}^{2}=\mathbb{E}\left(\left\langle Y_{t}, x^{*}\right\rangle^{2}\right) \\
=\left\|\Phi_{H}^{\prime}\left(I-\partial^{\prime} L^{\prime}\right)^{-1}\left(\delta_{t} \otimes x^{*}\right)\right\|_{L^{2}([0, T] ; H)}^{2} \\
=\int_{0}^{t}\left\|B^{*}\left(I-S^{*}(T)\right)^{-1} S^{*}(t-s) x^{*}\right\|_{H}^{2} d s+\int_{t}^{T}\left\|B^{*}\left(I-S^{*}(T)\right)^{-1} S^{*}(T+t-s) x^{*}\right\|_{H}^{2} d s \\
=\int_{0}^{T}\left\|B^{*}\left(I-S^{*}(T)\right)^{-1} S^{*}(s) x^{*}\right\|_{H}^{2} d s \\
=\left\|\Phi_{H}^{\prime}\left(I-\partial^{\prime} L^{\prime}\right)^{-1}\left(\delta_{T} \otimes x^{*}\right)\right\|_{L^{2}([0, T] ; H)}^{2} \\
=\left\langle R_{T} x^{*}, x^{*}\right\rangle .
\end{aligned}
$$

From this we infer that $R_{t}=R_{T}$. Moreover by (3.2),

$$
\begin{aligned}
\left\langle R_{T} x^{*}, x^{*}\right\rangle & =\int_{0}^{T}\left\|B^{*}\left(I-S^{*}(T)\right)^{-1} S^{*}(s) x^{*}\right\|_{H}^{2} d s \\
& =\int_{0}^{T}\left\|B^{*} S^{*}(s)\left(\left(I-S^{*}(T)\right)^{-1} x^{*}\right)\right\|_{H}^{2} d s \\
& =\left\langle Q_{T}\left(I-S^{*}(T)\right)^{-1} x^{*},\left(I-S^{*}(T)\right)^{-1} x^{*}\right\rangle .
\end{aligned}
$$

This shows that $R_{T}=(I-S(T))^{-1} Q_{T}\left(I-S^{*}(T)\right)^{-1}$.

Because a centred Gaussian measure is determined by its covariance operator, the first equality in Lemma 4.1 implies:

Corollary 4.2. For all $t, s \in[0, T]$, the laws of $Y_{s}$ and of $Y_{t}$ are the same.

Next we compare the laws of $Y_{T}$ and $X_{T}$. We start by presenting two sets of sufficient conditions for equality of the associated reproducing kernels. 
Proposition 4.3. Suppose that there exists a bounded linear operator $S_{H}(T)$ on $H$ such that

$$
B \circ S_{H}(T)=S(T) \circ B
$$

and assume that $I-S_{H}(T)$ is invertible in $H$. Then, as subsets of $E$, we have $G_{T}=H_{T}$. Proof: The assumptions imply that

$$
B^{*} \circ\left(I-S^{*}(T)\right)^{-1}=\left(I-S_{H}^{*}(T)\right)^{-1} \circ B^{*} .
$$

Hence, for all $x^{*} \in E^{*}$ we have

$$
\begin{aligned}
\left\langle R_{T} x^{*}, x^{*}\right\rangle & =\int_{0}^{T}\left\|B^{*} S^{*}(s)\left(\left(I-S^{*}(T)\right)^{-1} x^{*}\right)\right\|_{H}^{2} d s \\
& =\int_{0}^{T}\left\|B^{*}\left(\left(I-S^{*}(T)\right)^{-1} S^{*}(s) x^{*}\right)\right\|_{H}^{2} d s \\
& =\int_{0}^{T} \|\left(\left(I-S_{H}^{*}(T)\right)^{-1} B^{*} S^{*}(s) x^{*} \|_{H}^{2} d s\right. \\
& \leqslant\left\|\left(I-S_{H}^{*}(T)\right)^{-1}\right\|^{2} \int_{0}^{T}\left\|B^{*} S^{*}(s) x^{*}\right\|_{H}^{2} d s \\
& =\left\|\left(I-S_{H}^{*}(T)\right)^{-1}\right\|^{2}\left\langle Q_{T} x^{*}, x^{*}\right\rangle .
\end{aligned}
$$

This implies that $H_{T} \subset G_{T}$. Similarly, for all $x^{*} \in E^{*}$ we have

$$
\begin{aligned}
\left\langle Q_{T} x^{*}, x^{*}\right\rangle & =\int_{0}^{T}\left\|B^{*} S^{*}(s) x^{*}\right\|_{H}^{2} d s \\
& =\int_{0}^{T}\left\|\left(I-S_{H}^{*}(T)\right) B^{*} S^{*}(s)\left(I-S^{*}(T)\right)^{-1} x^{*}\right\|_{H}^{2} d s \\
& \leqslant\left\|I-S_{H}^{*}(T)\right\|^{2} \int_{0}^{T}\left\|B^{*} S^{*}(s)\left(I-S^{*}(T)\right)^{-1} x^{*}\right\|_{H}^{2} d s \\
& =\left\|I-S_{H}^{*}(T)\right\|^{2}\left\langle R_{T} x^{*}, x^{*}\right\rangle,
\end{aligned}
$$

from which we infer the opposite inclusion $G_{T} \subset H_{T}$.

Proposition 4.4. Suppose that $S(T)$ maps $G_{T}$ into itself and assume that the restriction of $I-S(T)$ to $G_{T}$ is invertible in $G_{T}$. Then, as subsets of $E$, we have $G_{T}=H_{T}$.

Proof: Denote by $S_{G_{T}}(T)$ the restriction of the operator $S(T)$ to $G_{T}$. The assumptions imply that $(I-S(T))^{-1}$ maps $G_{T}$ into itself, and that the restriction to $G_{T}$ coincides with the inverse of $I-S_{G_{T}}(T)$.

Let $i_{T}$ denote the inclusion map from $G_{T}$ into $E$. By general facts about reproducing kernels (cf. [DZ, Appendix B] or [Ne, Section 1]), we have the factorization $Q_{T}=i_{T} i_{T}$. Denoting by $S_{G_{T}}(T)$ the restriction of $S(T)$ to $G_{T}$, it follows from the identity $i_{T}^{*} \circ S^{*}(T) x^{*}=S_{G_{T}}^{*}(T) \circ i_{T}^{*} x^{*}$ that

$$
\left(I-S_{G_{T}}^{*}(T)\right)^{-1} i_{T}^{*} x^{*}=i_{T}^{*}\left(I-S^{*}(T)\right)^{-1} x^{*} .
$$


Hence, for all $x^{*} \in E^{*}$ we have

$$
\begin{aligned}
\left\langle R_{T} x^{*}, x^{*}\right\rangle & =\left\langle Q_{T}\left(I-S^{*}(T)\right)^{-1} x^{*},\left(I-S^{*}(T)\right)^{-1} x^{*}\right\rangle \\
& =\left\|i_{T}^{*}\left(I-S^{*}(T)\right)^{-1} x^{*}\right\|_{G_{T}}^{2} \\
& =\left\|\left(I-S_{G_{T}}^{*}(T)\right)^{-1} i_{T}^{*} x^{*}\right\|_{G_{T}}^{2} \\
& \leqslant\left\|\left(I-S_{G_{T}}^{*}(T)\right)^{-1}\right\|^{2}\left\|i_{T}^{*} x^{*}\right\|_{G_{T}}^{2} \\
& =\left\|\left(I-S_{G_{T}}^{*}(T)\right)^{-1}\right\|^{2}\left\langle Q_{T} x^{*}, x^{*}\right\rangle .
\end{aligned}
$$

This gives the inclusion $H_{T} \subset G_{T}$. Similarly, for all $x^{*} \in E^{*}$ we have

$$
\left\langle Q_{T} x^{*}, x^{*}\right\rangle \leqslant\left\|I-S_{G_{T}}^{*}(T)\right\|^{2}\left\langle R_{T} x^{*}, x^{*}\right\rangle .
$$

from which we infer the opposite inclusion $G_{T} \subset H_{T}$.

The assumptions of Proposition 4.4 are satisfied if $G_{T}$ is an interpolation space between $D(A)$ and $E$. In particular, they are satisfied in the situation when $H=E$ and $\{S(t)\}_{t \geqslant 0}$ is an analytic semigroup on $E$ : in this case we have $G_{T}=D_{A}\left(\frac{1}{2}, 2\right)$ [DZ, Appendix B].

We also mention the following sufficient condition for $S(T)$-invariance of $G_{T}$ [Ne, Lemma 1.10]: $S(T)$ maps $G_{T}$ into itself if there for all $t \in[0, T]$ there exists a bounded linear operator $S_{H}(t)$ such that $B \circ S_{H}(t)=S(t) \circ B$ and

$$
\int_{0}^{T}\left\|S_{H}(t)\right\| d t<\infty
$$

This gives a link between the conditions of Propositions 4.3 and 4.4.

Next we investigate under what conditions the laws of $Y_{T}$ and $X_{T}$ are equivalent.

Theorem 4.5. Suppose that $S(T)$ maps $G_{T}$ into itself and that the restriction of $S(T)$ to $G_{T}$ is Hilbert-Schmidt. Assume moreover that the restriction of $I-S(T)$ to $G_{T}$ is invertible in $G_{T}$. Then the laws of $Y_{T}$ and $X_{T}$ are equivalent.

Proof: Denote by $V_{T}: H_{T} \rightarrow G_{T}$ the map defined by

$$
V_{T}\left(R_{T} x^{*}\right)=Q_{T} x^{*}, \quad x^{*} \in E^{*} .
$$

By Proposition 4.4 we have $G_{T}=H_{T}$ as subsets of $E$, and therefore, by general facts concerning reproducing kernel spaces, the map $V_{T}$ is well-defined and extends to an isomorphism of $H_{T}$ onto $G_{T}$. Then by the closed graph theorem, as an operator on $G_{T}, V_{T}$ is an isomorphism as well. Choose a sequence $\left(x_{n}^{*}\right)$ in $E^{*}$ such that $\left(h_{n}\right)$, with $h_{n}=R_{T} x_{n}^{*}$, 
is an orthonormal basis for $G_{T}$. Then,

$$
\begin{aligned}
\sum_{n}\left\|\left(I-V_{T}\right) h_{n}\right\|_{G_{T}}^{2} & =\sum_{n}\left\|\left(I-V_{T}\right) R_{T} x_{n}^{*}\right\|_{G_{T}}^{2} \\
& =\sum_{n}\left\|\left(R_{T}-Q_{T}\right) x_{n}^{*}\right\|_{G_{T}}^{2} \\
& =\sum_{n}\left\|(I-S(T))^{-1} Q_{T}\left(I-S^{*}(T)\right)^{-1} x_{n}^{*}-Q_{T} x_{n}^{*}\right\|_{G_{T}}^{2} \\
& \left.=\sum_{n} \|\left(I-S_{G_{T}}(T)\right)^{-1}\left(I-S_{G_{T}}^{*}(T)\right)\right)^{-1} Q_{T} x_{n}^{*}-Q_{T} x_{n}^{*} \|_{G_{T}}^{2} \\
& =\sum_{n}\left\|\left(I-\left(I-S_{G_{T}}(T)\right)\left(I-S_{G_{T}}^{*}(T)\right)\right) h_{n}^{*}\right\|_{G_{T}}^{2} .
\end{aligned}
$$

This shows that $I-V_{T}$ is an Hilbert-Schmidt operator on $G_{T}$ if and only if the operator

$$
I-\left(I-S_{G_{T}}(T)\right)\left(I-S_{G_{T}}^{*}(T)\right)=S_{G_{T}}(T)+S_{G_{T}}^{*}(T)-S_{G_{T}}(T) S_{G_{T}}^{*}(T)
$$

is Hilbert-Schmidt on $G_{T}$.

Now by assumption, $S_{G_{T}}(T)$ is Hilbert-Schmidt on $G_{T}$, and therefore we conclude that $I-V_{T}$ is Hilbert-Schmidt on $G_{T}$. The equivalence of the measures $\nu_{T}$ and $\mu_{T}$ now follows from the Feldman-Hajék theorem.

As an illustration we consider the case of a self-adjoint diagonal generator $A$ :

Example 4.6. Let $E$ be a separable real Hilbert space. Suppose that $A$ is a selfadjoint operator in $E$ such that for some orthonormal basis $\left\{e_{j}\right\}_{j \in \mathbb{N}}$ in $E$ and some sequence of real numbers $\lambda_{j}$ one has

$$
A e_{j}=\lambda_{j} e_{j}, \quad j \in \mathbb{N} .
$$

We further let $H=E$. From Proposition 4.3 (or from Proposition 4.4 and the remarks following it) it is immediately clear that $G_{T}=H_{T}$. A simple computation gives, for $x=\sum x_{j} e_{j}$,

$$
\|x\|_{G_{T}}^{2}=\sum_{j=1}^{\infty} \frac{1}{2 \lambda_{j}}\left(1-e^{-2 \lambda_{j} T}\right)^{-1}\left|x_{j}\right|^{2}
$$

and

$$
\|x\|_{H_{T}}^{2}=\sum_{j=1}^{\infty}\left(1-e^{-\lambda_{j} T}\right)^{2} \frac{1}{2 \lambda_{j}}\left(1-e^{-2 \lambda_{j} T}\right)^{-1}\left|x_{j}\right|^{2} .
$$

Hence by the Feldman-Hajék theorem, the measure $\mu_{T}$ and $\nu_{T}$ are equivalent iff

$$
\sum_{j}\left|1-\left(1-e^{-\lambda_{j} T}\right)^{2}\right|^{2}=\sum_{j}\left(2 e^{-\lambda_{j} T} e^{-\lambda_{j} T}-e^{-2 \lambda_{j} T}\right)=\sum_{j} e^{-2 \lambda_{j} T}<\infty .
$$

This holds if and only if $S(T)$ is a Hilbert-Schmidt operator on $E$. Since $Q_{T}^{1 / 2}$ is an unitary isomorphism of $E$ onto $G_{T}$ commuting with $S(T)$, this is equivalent to $S_{G_{T}}(T)$ being Hilbert-Schmidt in $G_{T}$.

Acknowledgement - Part of this research was carried out while the first named author visited the Department of Mathematics at the University of Delft. He would like to thank the members of the department for their hospitality. 


\section{References}

[ABB] S. Albeverio, A.M. Boutet de Monvel-Berthier, and Z. Brzeźniak, The trace formula for Schrödinger operators from infinite dimensional oscillatory integrals, Math. Nachr. 182 (1996), 21-65.

[AH] S. Albeverio And R. Höegh-Krohn, Homogeneous random fields and statistical mechanics, J. Funct. Anal. 19 (1975), 242-272.

[Bi] J.M. Bismut, The Atiyah-Singer theorems: a probabilistic approach. I. The index theorem, J. Funct. Anal. 57 (1984), 56-99; II. The Lefschetz fixed point formulas, J. Funct. Anal. 57 (1984), 329-348.

[BN] Z. BrzeŹniak And J.M.A.M. VAn NeERven, Stochastic convolution in separable Banach spaces and the stochastic linear Cauchy problem, submitted for publication.

[DTb] G. Da Prato And L. Tubaro, Some results on periodic measures for differential stochastic equations with additive noise, Dynamic Systems Appl. 1 (1992), 131-139.

[DTd] G. Da Prato and C. Tudor, Periodic and almost periodic solutions for semilinear stochastic equations, Stochastic Anal. Appl. 13 (1995), 13-33.

[DZ] G. Da Prato And J. ZabczyK, "Stochastic Equations in Infinite Dimensions", Encyclopedia of Mathematics and Its Applications, Vol. 44, Cambridge University Press, Cambridge, 1992.

[Gr] G. Greiner, Perturbing the boundary conditions of a generator, Houston J. Math. 13 (1987), 213-229.

[JL] J. D. S. Jones And R. LÉAndRE, A stochastic approach to the Dirac operator over the free loop space, Tr. Mat. Inst. Steklova 217 (1997), Prostran. Petel i Gruppy Diffeomorf., 258-287.

[KL] A. Klein and L.J. Landau, Periodic Gaussian Osterwalder-Schrader positive processes and the two-sided Markov property on the circle, Pac. J. Math. 94 (1981), $341-367$.

[Lu] A. LunARdi, "Analytic Semigroups and Optimal Regularity in Parabolic Problems", Progress in Nonlinear Differential Equations and their Applications Vol. 16, Birkhäuser Verlag, Basel, 1995.

[Ne] J.M.A.M. van NeERven, Nonsymmetric Ornstein-Uhlenbeck semigroups in Banach spaces, J. Funct. Anal. 155 (1998), 495-535.

[No] J.R. NorRis, Ornstein-Uhlenbeck processes indexed by the circle, Ann. Probab. 26 (1998), 465-478.

[Pa] A. PAZY, "Semigroups of Linear Operators and Applications to Partial Differential Equations," Springer-Verlag, 1983

[VTC] N.N. Vakhania, V.I. Tarieladze, and S.A. Chobanyan, "Probability Distributions on Banach Spaces", D. Reidel Publishing Company, Dordrecht-Boston-Lancaster-Tokyo, 1987. 